\title{
Overwien, Bernd
}

\section{Wie politisch ist politische Bildung? Kontroversen in der politischen Bildung seit den siebziger Jahren}

Thole, Friederike [Hrsg.]; Wedde, Sarah [Hrsg.]; Kather, Alexander [Hrsg.]: Über die Notwendigkeit der Historischen Bildungsforschung. Wegbegleiter*innenschrift für Edith Glaser. Bad Heilbrunn : Verlag Julius Klinkhardt 2021, S. 115-124

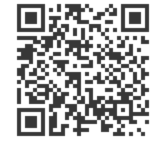

\section{Quellenangabe/ Citation:}

Overwien, Bernd: Wie politisch ist politische Bildung? Kontroversen in der politischen Bildung seit den siebziger Jahren - In: Thole, Friederike [Hrsg.]; Wedde, Sarah [Hrsg.]; Kather, Alexander [Hrsg.]: Über die Notwendigkeit der Historischen Bildungsforschung. Wegbegleiter*innenschrift für Edith Glaser. Bad Heilbrunn : Verlag Julius Klinkhardt 2021, S. 115-124 - URN: urn:nbn:de:0111-pedocs-233420 - DOI: 10.25656/01:23342

\section{http://nbn-resolving.org/urn:nbn:de:0111-pedocs-233420}

http://dx.doi.org/10.25656/01:23342

in Kooperation mit / in cooperation with:

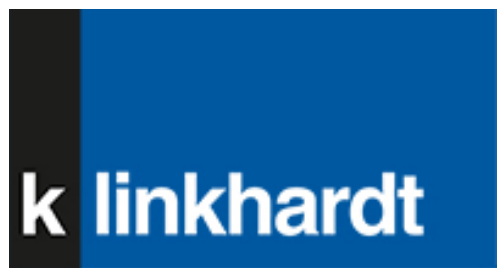

http://www.klinkhardt.de

\section{Nutzungsbedingungen}

Dieses Dokument steht unter folgender Creative Commons-Lizenz: http://creativecommons.org/licenses/by-nc-sa/4.0/deed.de - Sie dürfen das Werk bzw. den Inhalt unter folgenden Bedingungen vervielfältigen, verbreiten und öffentlich zugänglich machen sowie Abwandlungen und Bearbeitungen des Werkes bzw. Inhaltes anfertigen: Sie müssen den Namen des Autors/Rechteinhabers in der von ihm festgelegten Weise nennen. Dieses Werk bzw. der Inhalt darf nicht für kommerzielle Zwecke verwendet werden. Die neu entstandenen Werke bzw. Inhalte dürfen nur unter Verwendung von Lizenzbedingungen weitergegeben werden, die mit denen dieses Lizenzvertrages identisch oder vergleichbar sind.

Mit der Verwendung dieses Dokuments erkennen Sie die Nutzungsbedingungen an.

\section{Terms of use}

This document is published under following Creative Commons-License: http://creativecommons.org/licenses/by-nc-sa/4.0/deed.en - You may copy, distribute and transmit, adapt or exhibit the work in the public and alter, transform or change this work as long as you attribute the work in the manner specified by the author or licensor. You are not allowed to make commercial use of the work. If you alter, transform, or change this work in any way, you may distribute the resulting work only under this or a comparable license.

By using this particular document, you accept the above-stated conditions of use.

\section{Kontakt / Contact:}

\section{peDOCs}

DIPF | Leibniz-Institut für Bildungsforschung und Bildungsinformation

Informationszentrum (IZ) Bildung

E-Mail: pedocs@dipf.de

Internet: www.pedocs.de

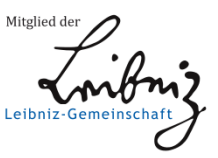




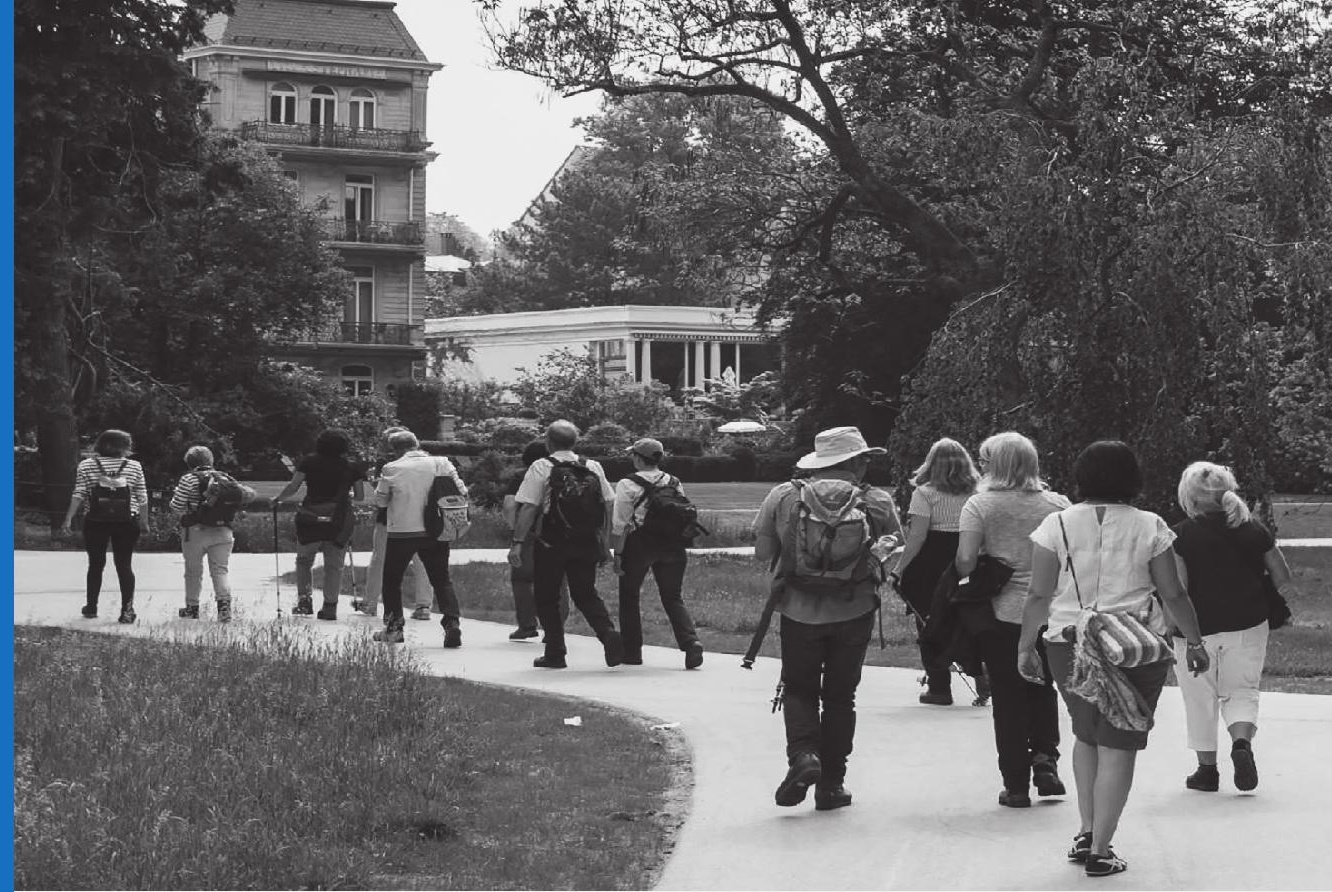

Friederike Thole

Sarah Wedde Alexander Kather

(Hrsg.)

Über die Notwendigkeit der Historischen Bildungsforschung

Wegbegleiter*innenschrift für Edith Glaser 


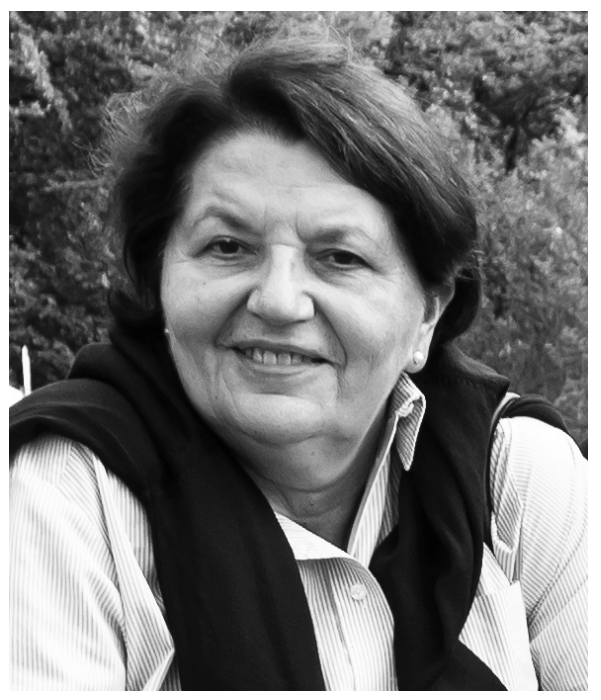

Edith Glaser ist seit 2006 Professorin für Historische Bildungsforschung an der Universität Kassel. Ihre Forschungsschwerpunkte sind die Disziplingeschichte der Erziehungswissenschaft, die historische Frauenund Geschlechterforschung sowie die geschichtliche Perspektive auf Bildungspolitik und Bildungsberatung. 


\section{Friederike Thole Sarah Wedde Alexander Kather (Hrsg.)}

\section{Über die Notwendigkeit der Historischen Bildungsforschung}

Wegbegleiter*innenschrift für Edith Glaser 
Dieser Titel wurde in das Programm des Verlages mittels eines Peer-Review-Verfahrens aufgenommen. Für weitere Informationen siehe www.klinkhardt.de.

Bibliografische Information der Deutschen Nationalbibliothek Die Deutsche Nationalbibliothek verzeichnet diese Publikation in der Deutschen Nationalbibliografie; detaillierte bibliografische Daten sind im Internet abrufbar über http://dnb.d-nb.de.

2021.n. () by Julius Klinkhardt.

Foto Umschlagseite 1: () Achim Heinrichs.

Druck und Bindung: AZ Druck und Datentechnik, Kempten.

Printed in Germany 2021.

Gedruckt auf chlorfrei gebleichtem alterungsbeständigem Papier.

(c) (i)(2) Die Publikation (mit Ausnahme aller Fotos, Grafiken und Abbildungen) ist veröffent-

licht unter der Creative Commons-Lizenz: CC BY-NC-SA 4.0 International

https://creativecommons.org/licenses/by-nc-sa/4.0/

ISBN 978-3-7815-5914-1 digital doi.org/10.35468/5914

ISBN 978-3-7815-2479-8 


\section{Inhaltsverzeichnis}

Friederike Thole, Sarah Wedde und Alexander Kather

Über die Notwendigkeit der Historischen Bildungsforschung -

Einführung in die Wegbegleiter*innenschrift für Edith Glaser

\section{Teil I}

Einblicke in ausgewählte Arbeitsbereiche der Historischen Bildungsforschung

Sarah Wedde und Friederike Thole

Historische Bildungsforschung schreibt Wissensgeschichte

\section{Ulrich Herrmann}

Die Bedeutung des Gemeinten im Gesagten.

Das Werkzeug des Historikers: Philologie und Hermeneutik -

am Beispiel von Wilhelm Flitners Veröffentlichungen in der NS-Zeit

Christian Bluhm und Markus Wochnik

Gender in der beruflichen Bildung - Entwicklung einer „Kategorie“

und einer zeitgemäßen Auseinandersetzung

\section{Pia Schmid}

Schule, Lehrerinnen und Lehrer in Autobiographien

politisch engagierter Frauen - 1850 bis 1910

Alexander Kather

„Wo die Quelle des Wissens ununterbrochen fließt“ -

Auslandsaufenthalte von Fremdsprachenlehrenden

aus (fach-)wissensgeschichtlicher Perspektive

Elke Kleinau

Children Born of War in der deutschen Nachkriegsgesellschaft -

Pädagogischer Diskurs und biografische Erzählungen 


\section{Teil II}

\section{Historische Bildungsforschung im inner- und interdisziplinären Kontext}

Ralf Mayer

,Zur Aufgabe des Erinnerns ‘ - im Spannungsfeld

von Orientierungsanspruch und Erinnerung

Werner Thole und Max-Ferdinand Zeterberg

Entdecken, Vergessen und Erinnern.

Über das ambivalente Rendezvous der Erziehungswissenschaft mit

der Sozialpädagogik bei Klaus Mollenhauer

Bernd Overwien

Wie politisch ist politische Bildung?

Kontroversen in der politischen Bildung seit den siebziger Jahren

Annedore Prengel

Anna und Maria mit dem Buch -

eine imaginierte pädagogische Beziehung

Friederike Heinzel und Julian Storck-Odabasi

100 Jahre Grundschule - Konzepte des Anfangsunterrichts

\section{Isabelle Naumann}

Von der Meritentafel zur Verhaltensampel -

Lob und Strafe in der Grundschule

Winfried Speitkamp

Vom Bauhaus lernen:

Das Staatliche Bauhaus von 1919 und die Universität der Zukunft 161

Verzeichnis der Autor*innen 


\section{Bernd Overwien}

\section{Wie politisch ist politische Bildung? Kontroversen in der politischen Bildung seit den siebziger Jahren}

Politischer Bildung, die als historisch-politische Bildung in engem Zusammenhang mit historischer Bildungsforschung steht, geht es als Teil gesellschaftlicher Allgemeinbildung um politische, soziale, wirtschaftliche und auch kulturelle $\mathrm{Zu}$ sammenhänge. Im Mittelpunkt steht das ethisch-moralische Urteilen genauso wie politisches Handeln (vgl. DVPB, 2014). Politische Bildung trägt zur Herausbildung entsprechenden Wissens und Könnens bei. Sie leistet einen Beitrag zur Allgemeinbildung, indem sie es den Lernenden im besten Fall ermöglicht, an ihrer „Selbstbestimmungs-, Mitbestimmungs- und Solidaritätsfähigkeit“ zu arbeiten (Klafki, 2005, S. 2). Aus dem Geist der Aufklärung ${ }^{1}$ heraus versteht sich politische Bildung bis heute als Beitrag zur Selbstbefreiung mit dem Ziel größtmöglicher Mündigkeit und Emanzipation, die Klaus-Peter Hufer (2013, S. 24f.) von einer eher individuellen Entwicklung insofern löst, indem er emanzipatorische Ziele mit Solidarität verknüpft. Letztendlich muss es auch um die Frage gehen, in welche Strukturen und Zwänge Menschen eingebunden sind und wie diese sie hindern, Zugänge zu politischen Feldern zu finden (vgl. Eis, 2013, S. 74f.). Politische Mündigkeit darf nicht verengt werden - zugespitzt ausgedrückt - auf eine in Herrschaftsstrukturen eingebundene kontrollierte Selbstoptimierung. Emanzipatorisch ist politische Bildung dann, wenn sie die angedeuteten Strukturen mit thematisiert, so subtil und komplex sie auch sein mögen. Es soll um die Entwicklung der Fähigkeit gehen, in gesellschaftlichen Zusammenhängen zu denken. Peter Henkenborg (2013) fügt eine weitere Perspektive hinzu. Anschließend an Adorno geht er davon aus, dass das Individuum sich vollständig erst in einer gerechten, menschlichen Gesellschaft finde. Auf dem Weg dorthin bedürfe es der politischen Bildung als Ort kritischer Gegenöffentlichkeit, verbunden mit Gesellschaftskritik und einem Denken in Dimensionen von Gesellschaftsveränderung. Diese Perspektive sei in der Politikdidaktik heute „weitgehend verschwunden“ (S. 111).

1 Wenn hier von der Periode der Aufklärung die Rede ist, muss allerdings auch deren Begrenztheit mit erwähnt werden, etwa ihr Eurozentrismus, Rechtfertigungen des Kolonialismus, Sexismus (vgl. Dhawan, 2016). 


\section{Politische Bildung und Kompetenzen}

Letztendlich sollte es in der politischen Bildung insgesamt nach wie vor um gesellschaftliche Schlüsselkompetenzen gehen, die von Wolfgang Klafki „epochaltypische Schlüsselprobleme“ genannt wurden. Es geht dabei um „Strukturprobleme von gesamtgesellschaftlicher, meistens sogar übernationaler bzw. weltumspannender Bedeutung [...], die gleichwohl jeden einzelnen zentral betreffen." Sie sind „epochaltypisch“, wenn es sich dabei „um einen in die Zukunft hinein wandelbaren Problemkanon handelt" (Klafki, 1996, S. 60). Diese Schlüsselprobleme enthalten ,inhaltsbezogene und kommunikationsbezogene Komponenten“, wobei vor allem die Befähigung zum „vernetzenden Denken“ eine besondere Rolle spielt (ebd., S. 63). Epochaltypische Schlüsselprobleme sind nach Klafki (ebd., S. 56ff.) unter anderem die ökologische Frage, die Friedensfrage, die gesellschaftlich verursachte Ungleichheit oder die Gefahren und Möglichkeiten der Digitalisierung. Dies bezieht er auf die Aufgaben der Allgemeinbildung.

Unter dem Einfluss des ,Pisa-Schocks' Anfang der 2000er-Jahre kam es zu einem Paradigmenwechsel von den Lernzielen als Grundlage einer Input-Steuerung hin zu Kompetenzen als geforderte Ergebnisse von Lernprozessen. Historisch immerhin eng verknüpft mit dem Dreischritt Sehen - Beurteilen - Handeln (vgl. George \& Hilligen, 1971), entwickelte die Gesellschaft für Politikdidaktik und politische Jugend- und Erwachsenenbildung (im Folgenden mit GPJE abgekürzt) als wissenschaftliche Fachgesellschaft der Politikdidaktik, ein Kompetenzkonzept mit den drei Kompetenzbereichen der methodischen Kompetenzen, der politischen Urteilsfähigkeit und der politischen Handlungsfähigkeit. In einer sehr offenen Formulierung wurde dazu die Notwendigkeit eines ,konzeptionellen Deutungswissens" betont (GPJE, 2004). Bemerkenswert ist, dass es im deutschsprachigen Raum einen sehr breiten Konsens darüber gibt, dass die Förderung politischer Urteils- und Handlungsfähigkeit zu den wesentlichen Aufgaben politischer Bildung zählt (vgl. Sander, 2014, S. 118f.).

Die Grundstruktur des Kompetenzkonzeptes findet sich heute in vielen Curricula der Bundesländer. Unterschiede lassen sich beim Umgang mit der Kategorie „Wissen“ beobachten. An dieser Frage entzündete sich auch ein gravierender Konflikt innerhalb der politischen Bildung, indem es einerseits darum geht, ob diese sich enger an die Politikwissenschaft bindet (vgl. Detjen et al., 2012) oder eher ein sozialwissenschaftliches Integrationsfach konturiert, das auch soziologische, ökonomische und juristische Dimensionen in den Blick nimmt (vgl. Autorengruppe Fachdidaktik, 2011). Unterschiede zeigen sich andererseits am Umgang mit dem den Kompetenzen zugrunde liegenden Wissen. So grenzen die einen ein engeres Verständnis von fachlichem Wissen gegen „Fehlkonzepte“ ab, während die andere Seite die Pluralität wissenschaftlichen Wissens betont und die Vielfalt der daraus entstehenden Schüler*innenvorstellungen. Die eine Seite fügt sich 
stärker an kognitionspsychologische Vorstellungen an, lehnt bildungstheoretische Bezüge explizit ab (vgl. Detjen et al., 2012, S. 11) und zielt auf eine durchgängige Testbarkeit von Kompetenzen, die allerdings danach nur kognitiv abbildbar sind. Die andere Seite betont den Zusammenhang von Bildung und Kompetenz (vgl. Sander, 2014, S. 120f.).

\section{Kontroverse Sichtweisen zur schulischen Umsetzung der politischen Handlungsfähigkeit}

Kompetenz wird bekanntlich erst in ihrer Performanz, das heißt in ihrer praktischen Anwendung als Fähigkeit sichtbar. Insofern stellt sich einmal die Frage, wie ein kompetenzorientierter Unterricht aussehen kann und dann, wie die erreichten Kompetenzen abbildbar sind. Für Autor*innen, die einem eher kognitiv geprägten Kompetenzverständnis folgen, ist die Lage klar. Urteils- und Handlungsfähigkeit ist nur jeweils von ihrer kognitiven Seite aus im Unterricht bearbeitbar (vgl. Detjen et al., 2012, S. 12, 14). Wolfgang Sander sieht hier mit Recht eine Reduktion des theoretischen Denkens in der Politikdidaktik (vgl. Sander, 2013, S. 116). Ein eng mit politischer Bildung verbundenes unterrichtliches Arbeiten, wie etwa ein Beobachten im Feld, das Durchführen kleinerer Interviews, die Analyse von Medien unter Fragestellungen der politischen Bildung oder auch die Durchführung von kleineren Studien werden hier zu fachunspezifischen Aktivitäten erklärt (vgl. Sander, 2013, S. 117). Zwar ist unbenommen, dass gerade hier auch die Möglichkeit eines fächerübergreifenden Arbeitens naheliegt, dies wird aber durch zu enge Didaktikvorstellungen nicht gerade gefördert. Politisches Handeln, das auf Entscheidungen auf den Ebenen des politischen Systems gerichtet ist, sei im Politikunterricht nicht möglich. Politikunterricht könne „bestenfalls" auf die damit verbundenen Prozesse vorbereiten (Detjen et al., 2012, S. 66). Sibylle Reinhardt (2017) hingegen sieht die Institution Schule durchaus auch als politisches Aktionsfeld und damit als Feld realen Handelns. Politische Bildung müsse politisches Handeln betreffen, Bürgeraktivität beschränke sich sonst auf Reflexion. Sie sieht dabei einerseits aber das Problem eines Aktionismus und andererseits die Gefahr einer Überwältigung. Der Beutelsbacher Konsens sei zwar aktuell und richtig, Lehrkräfte seien aber nicht neutral und sollten sich auch nicht so gerieren, da sie sich unglaubwürdig machten, wenn sie sich für unfähig zu einer politischen Stellungnahme erklärten. Die politische Aktion aus dem Unterricht heraus sei aber problematisch, so Reinhardt, weil sowohl die Haltung der Lehrkraft, als auch die Gruppendynamik einer Lerngruppe Überwältigungspotential habe. 
Im Bereich der Demokratiebildung, der eigentlich viel enger mit politischer Bildung zusammengedacht werden müsste, als dies bisher geschieht, erfreut sich ein Lernen für ein zivilgesellschaftliches Engagement breiter Unterstützung. Service-Learning ist dort einerseits „Dienst am Gemeinwohl“ (Sliwka, 2004, S. 5) und anderseits soll in Projekten etwas über bürgerschaftliches Engagement und auch politisches Handeln gelernt werden. Im Mittelpunkt steht dabei die direkte Erfahrung. Aus der Perspektive kritischer politischer Bildung sieht Alexander Wohnig (2017) große Diskrepanzen zwischen der Beurteilung von Projekten des Demokratie-Lernens durch den politikdidaktischen „Mainstream“ und deren Sicht auf Aktivitäten im Rahmen eines Service-Learning. Solange es um ein soziales Lernen gehe, fänden sich positive Beurteilungen, komme es aber zu politischem Handeln, werde auf die Überwältigungsgefahr verwiesen (ebd., S. 136f.). Abgesehen von der auch spannenden Frage, wie soziales und politisches Lernen zusammenhängen, wird hier der potential fruchtbare Kontext von Handeln und Lernen abgeschnitten, wenn es um für die Politik relevantes Lernen geht. Dabei wird an vielen anderen Stellen im Bildungskontext darauf verwiesen, wie produktiv Verbindungen zwischen formalem und informellem Lernen sein können (vgl. Overwien, 2005). Gerade auch wenn der kommunale Kontext gesehen wird, können hier Schüler*innen konkrete Selbstwirksamkeitserfahrungen sammeln, wenn sie sich aktiv einbringen (vgl. Wohnig, 2018). In der Politikdidaktik kann damit an nach wie vor aktuellen Hinweisen aus den 1970erJahren angesetzt werden. Sowohl Rolf Schmiederer (1971) als auch Hermann Giesecke (1971) sahen damals die Lerngelegenheiten, die sich aus politischem Handeln und politischer Aktion ergeben. Neuerdings werden diese Debatten wieder produktiv aufgenommen (vgl. Wohnig, 2018; Kenner, 2021).

\section{Der Beutelsbacher Konsens und der Umgang mit sehr unterschiedlichen politischen Positionen}

Ein emanzipatorisches Verständnis politischer Bildung wird neuerdings massiv angegriffen. Rechtspopulisten behaupten, „das Volk“ zu vertreten und wenden sich gegen Grundprinzipen der Demokratie, wie das des kontroversen Umgangs mit politischen Problemen. Einer differenzierten Auseinandersetzung werden Behauptungen entgegengestellt. Ständig wird polarisiert in den Figuren eines „Wir" und „die Anderen“, in „gut oder böse“ und „richtig oder falsch“. Es wird behauptet, in Deutschland existiere keine Freiheit der Rede, gleichzeitig werden Verschwörungserzählungen und angstauslösende Katastrophenszenarien verbreitet (vgl. Hafeneger, 2017). 
Angesichts derartiger Angriffe müssen sich Akteur*innen der politischen Bildung verstärkt mit ihrem Auftrag und den einschlägigen Regeln auseinandersetzen (vgl. Mittnik et al., 2018). Es geht nicht um ein Zurückweichen, sondern um eine bewusste, starke und möglichst offensive Verteidigung demokratischer Werte. Dabei stehen nach wie vor eine wissenschaftlich gestützte Rationalität und eine offene Problemabwägung den angedeuteten Mythen und der Propaganda entgegen, die ja auch zum Wesen autoritärer Herrschaft gehören (vgl. Hafeneger, 2017, S. 51). Gleichzeitig muss sich politische Bildung und auch Demokratiebildung stärker als bisher mit den durch Politik ausgelösten Gefühlslagen beschäftigen. Dabei geht es um Ohnmacht, Angst, Wut und Verzweiflung, um Hass und Neid. Es geht aber auch um Hoffnung, Solidarität oder die Fähigkeit zur Empathie (vgl. Besand et al., 2019).

Wichtig ist dabei der Beutelsbacher Konsens, der in den 1970er Jahren formuliert wurde, zu Zeiten erheblicher politischer Auseinandersetzungen über die Ziele und Wege politischer Bildung. Innerhalb der politischen Bildung und ihrer Didaktik kam es in dieser Zeit zu einer Frontenbildung. Diese hat eng mit den politischen Entwicklungen dieser Zeit zu tun. Gegen Ende der 1960er Jahre wurde eine Entwicklung sichtbar, die sich schon vorher abzeichnete. Vor allem Studierende wehrten sich gegen überkommene Strukturen in den Universitäten und engagierten sich gesellschaftlich in der Bewegung gegen die Notstandsgesetzgebung und gegen den Vietnamkrieg. Gleichzeitig wurde ein Generationenkonflikt deutlich, der in Deutschland auch mit der nun offeneren Diskussion über die Verbrechen der Zeit des Nationalsozialismus zu tun hatte. Schließlich war es die Elterngeneration dieser "68er"-Studierenden, denen sich dann auch Schüler*innen und Lehrlinge anschlossen, die teils an den Verbrechen beteiligt waren oder die sie duldeten. Im Wissenschaftsbereich wurden die Schriften der Frankfurter Schule breit gelesen. Autoren wie Horkheimer, Adorno und Marcuse erreichten eine spürbare Wirkung. Die Protestbewegung war politisch breit gefächert. So wurde der Marxismus neu rezipiert, zumeist in scharfer Abgrenzung zur DDR. Auch unter Bezug auf Habermas wurde eine stärkere Demokratisierung der noch jungen Bundesrepublik Deutschland gefordert und zu Teilen auch erreicht. Die politische Bildung geriet nun in eine polarisierte öffentliche Diskussion um Lehrpläne, also um die Inhalte, die in der Schule zu verhandeln sein sollen. Links der politischen Mitte wurde Emanzipation und eine möglichst weitgehende Demokratisierung in den Vordergrund gestellt, im eher konservativen Lager sahen viele den Einzug des Klassenkampfes in die Schule kommen und betonten den Wert des rationalen Urteils. Aus heutiger Sicht ist es kaum noch vorstellbar, dass man sich damals um „Befähigung zu Selbst- und Mitbestimmung“ in der Gesellschaft als ein schulisches Ziel stritt. Damals wurden mit den damit verbundenen Fragen Wahlkämpfe ausgetragen (Sander, 2013, S. 138ff.; Gagel, 2005, S. 177ff.). 
Zwar wurde gesellschaftliche Reform in der Didaktik insgesamt positiv aufgenommen und ,Emanzipation' wurde zu einem weit geteilten Schlüsselbegriff der politischen Bildung, der mit dem Lernziel der Mündigkeit verbunden wurde. Gleichzeitig entwickelten sich auch Ansätze, die sich stärker auf die Kritische Theorie bezogen und sich auf neomarxistische Theoriekonzepte stützten. Zur selben Zeit meldeten sich verstärkt auch Autor*innen zu Wort, die sich im liberalen und konservativen politischen Spektrum verorteten. So waren die hier kurz skizzierten gesellschaftspolitischen Konfliktfelder auch in der politischen Bildung sichtbar. Während sich in der Bildungspolitik zum Teil größere Gräben auftaten, blieb in der Politikdidaktik immerhin die Grundlage für das gemeinsame Gespräch erhalten, wenn auch deutliche Kontroversen ausgetragen wurden. Um weiterhin gemeinsam arbeitsfähig bleiben zu können, kam es dann 1976 im schwäbischen Beutelsbach zu einer Tagung, während der die Kontroversen diskutiert wurden. Dabei schälte sich ein Minimalkonsens heraus, der als ,Beutelsbacher Konsens in die Bildungsgeschichte einging. Dieser enthält ein Überwältigungsverbot, ein Gebot der Kontroversität und ein Gebot der Orientierung an den Interessen der Schüler*innen (vgl. Sander, 2013, S. 218). Er ist seitdem ein kaum kritisierter Grundbestand des professionellen Selbstverständnisses der politischen Bildung. Der Beutelsbacher Konsens kann also als wichtiger Verständigungsschritt in einer besonderen historischen Konstellation gesehen werden. Er gewinnt heute an Bedeutung, weil er vor allem aus rechtspopulistischer Richtung als Neutralitätsgebot interpretiert wird.

Der Beutelsbacher Konsens ist aber kein Neutralitätsgebot. Lehrer*innen dürfen und sollen eigene politische Positionen haben. Lehrer*innen müssen allerdings immer darauf achten, dass bei den Schüler*innen nicht der Eindruck entstehe, die Meinung der Lehrkraft hätte bei künftigen Meinungsäußerungen ein höheres Gewicht als ihre eigenen Positionen. Eine professionell auf hohem Niveau arbeitende Lehrkraft begrüße ausdrücklich ein diverses Meinungsbild (vgl. Mittnik et al., 2018, S. 24; vgl. Oberle 2017)

Eine strikte Neutralität der Lehrperson könnte sogar ein völlig falsches Signal an die Schüler*innen senden. Frank Nonnenmacher (2011) hält „dieses Rollenvorbild, das von einer solchen Lehrperson gegeben wird, für höchst fatal. Es fördert die Tugend der Meinungslosigkeit, des Sich-Heraushaltens, des Nicht-Flagge-Zeigens." (S. 91) Vielmehr könnten Schüler*innen in der Lehrkraft insofern ein Vorbild wahrnehmen, als dass sie eine politisch interessierte, mitunter sogar aktive Person sehen, die sich mit dem Thema auseinandersetzt und aus den zur Verfügung stehenden Informationen eine individuelle Meinung entwickelt hat. Rollenvorbilder bieten jungen Menschen darüber hinaus auch eine Projektionsfläche, an der sie sich abarbeiten können.

Jahrelang etwas ausgeblendet blieb die Frage, wie es denn um Grenzen der Kontroversität bestellt sei und lange hat kaum jemand darüber gesprochen, dass das 
Grundgesetz und die Menschenrechte als normativer Hintergrund des Beutelsbacher Konsenses gesehen werden müssen. Möglicherweise galt dies als selbstverständlich. Tilmann Grammes (2014, S. 267) betont, dass das Kontroversprinzip keineswegs wertneutral oder relativistisch sei, sondern in einer normativen Pluralismustheorie begründet werde. Es gehört zur Professionalität im Lehrberuf, erfordert aber einen hohen Grad an Reflexivität.

\section{Ausblick}

Politische Bildung hat sich in Deutschland zwar in schulischen und außerschulischen Kontexten etabliert, hat aber leider viele Bezüge zur historisch-politischen Bildung verloren. Gleichzeitig wird es künftig darauf ankommen, stärkere Brücken hin zur eher erziehungswissenschaftlichen Demokratiepädagogik zu bauen. Politische Bildung kann sich dabei auch durch eine stärkere Lebensweltorientierung durchaus interessanter für junge Menschen gestalten. Dazu passen auch Kooperationen von Schulen mit außerschulischen Partnern. Auch sollten sich schulische Aktivitäten, auch jene der politischen Bildung, an Modellen der Schulentwicklung orientieren, wie sie im Konzept des „whole-school-approach“ englischsprachiger Länder zum Ausdruck kommen und wie es der „Orientierungsrahmen für den Lernbereich globale Entwicklung der Kultusministerkonferenz (KMK/ BMZ, 2016) empfiehlt. Der Orientierungsrahmen thematisiert die Integration globaler Themenstellungen und Fragen einer nachhaltigen Entwicklung in (fast) alle Unterrichtsfächer, in Schulentwicklung und Lehrer*innenbildung. Er enthält auch Vorschläge, wie die politische Bildung ihre viel zu lang verfolgte Engführung auf den nationalen Kontext überwinden kann (vgl. Overwien, 2017). Der oben genannte Orientierungsrahmen versteht sich als Beitrag zu einer „Bildung als Fundament zukunftsfähiger Entwicklung" (KMK/BMZ, 2016, S. 16). Wenn es allerdings um die Normativität nachhaltiger Entwicklung geht, stehen entsprechenden unterrichtlichen Handlungsoptionen der politischen Bildung oft auch Fehlinterpretationen des Beutelsbacher Konsens im Wege. Die Zielvorstellungen nachhaltiger Entwicklung können durchaus als Konkretisierung des Artikels 20a des Grundgesetzes gesehen werden. Sie lösen aber teils mit Blick auf den Beutelsbacher Konsens Befremden aus. Bildungs- und Kompetenzziele, die im Bereich von Emotionen, Werten und Ethik verortet sind, verstärkten diesen Eindruck oft (vgl. Overwien, 2016). Umwelt- und Entwicklungsfragen, aber auch viele andere Aspekte politischer Bildung, sind schließlich in der professionellen Handhabung von Gefühlslagen eine berufliche Herausforderung. Viele damit verbunden Fragestellungen lösen Ängste aus, wie Kinder- und Jugendstudien seit Jahren zeigen (vgl. Schneekloth \& Albert, 2010). Erst eine Enttabuisierung emotionaler Dimen- 
sionen politischer Diskurse ermöglicht aber einen rationalen Umgang damit. Das zeigen auch Umgehensweisen mit dem Phänomen des Klimawandels, der trotz aller immer wieder geschürten Zweifel inzwischen im Bewusstsein der breiten Bevölkerung angekommen ist. Hier liegen erhebliche Angstpotentiale, deren Bewältigungsstrategien einerseits als kollektiver Verdrängungsprozess gesehen werden kann, anderseits treibt viele Menschen die Hoffnung auf verbesserte Technologien um, die die Probleme lösen helfen sollen (vgl. Overwien, 2019; Hippe, 2018). Thorsten Hippe (2016, S. 204ff.) sieht hier die Notwendigkeit verstärkter Aufklärung einerseits. Anderseits sieht er - empirisch gestützt - Erfolgsmöglichkeiten in der Bildung auch bei einer stärkeren Fokussierung des Mensch-Natur-Verhältnisses, letztlich zur Förderung des Gefühls der Zugehörigkeit zur Natur. Entsprechende Reflexionen sollten nicht ausschließlich ethisch-rational erfolgen, sondern benötigten auch eine intuitive und ästhetisch-emotionale Verankerung, die mit Naturerfahrung verbunden sei.

Darüber hinaus muss unterstrichen werden, dass die wesentlichen Herausforderungen für die Gestaltung der Zukunft globaler Natur sind, das zeigen die Coronakrise 2020/21 genauso wie die anhaltende Klimakrise. Bewältigt werden die damit verbundenen komplexen Problemzusammenhänge nur in globaler Kooperation, die sich in einem Wirkungsgefüge von global und lokal bewegen muss. Mit der Agenda 2030 und den 17 Nachhaltigkeitszielen der Vereinten Nationen gibt es eine Plattform zur Bewältigung globaler Krisen. Mit einer Realisierung der Sustainable Development Goals (SDGs) ist eine "große Transformation“ von Wirtschaft und Gesellschaft verbunden, die der „Wissenschaftliche Beirat der Bundesregierung Globale Umweltveränderungen“" schon 2011 umrissen und gefordert hat (WBGU, 2011). Bildung spielt auf allen damit verbunden Feldern eine wichtige Rolle (vgl. Singer-Brodowski, 2016). Hierbei lässt sich politische Bildung kaum von einem entsprechenden Handeln trennen.

Abschließend muss allerdings darauf hingewiesen werden, dass politische Bildung als Schulfach gemessen an den Stundentafeln einen eher geringen Anteil schulischer Bildung darstellt. Gleichzeitig ist politische Bildung als Aufgabe der ganzen Schule, wie sie in allen Schulgesetzen der Länder steht, nicht genügend Praxis. Demokratiepädagogische Diskussionen der vergangenen Jahre, die sich ja immer wieder auf John Dewey beziehen, bieten Anschluss für gemeinsame Initiativen, zur Realisierung einer breiteren gesellschaftswissenschaftlichen Bildung an deutschen Schulen. 


\section{Literatur}

Autorengruppe Fachdidaktik (2011). Konzepte der politischen Bildung - eine Streitschrift. Schwalbach/Ts.: Wochenschau.

Besand, Anja, Overwien, Bernd \& Zorn, Peter (2019). Gefühle über Gefühle. Zum Verhältnis von Rationalität und Emotionalität in der politischen Bildung - eine Einführung. In Dies. (Hrsg.), Politische Bildung mit Gefühl. Bonn: BpB.

Detjen, Joachim, Massing, Peter, Richter, Dagmar \& Weißeno, Georg (2012). Politikkompetenz - ein Modell. Wiesbaden: Springer.

Dhawan, Nikita (2016). Die Aufklärung retten: Postkoloniale Interventionen. Zeitschrift für Politische Theorie 7(2), S. 249-255.

DVPB (2014). Politische Bildung für die Demokratie. Positionspapier der Deutschen Vereinigung für Politische Bildung. http://dvpb.de/wir-ueber-uns/positionen/positionspapier/

Eis, Andreas (2013). Mythos Mündigkeit - oder Erziehung zum funktionalen Subjekt. In Benedikt Widmaier \& Bernd Overwien (Hrsg.), Was heißt heute kritische politische Bildung? Schwalbach/Ts.: Wochenschau, S. 69-77.

George, Siegfried \& Hilligen, Wolfgang (1971). Sehen - beurteilen - handeln, 5./6. Schuljahr. Frankfurt a. M.: Cornelsen.

Giesecke, Hermann (1971). Didaktische Probleme des Lernens im Rahmen von politischen Aktionen. In Hermann Giesecke, Dieter Baake, Hermann Glaser, Theodor Ebert, Gernot Jochheim \& Brückner, Peter (Hrsg.), Politische Aktion und politisches Lernen. München: Juventa.

GPJE (Gesellschaft für Politikdidaktik und politische Jugend- und Erwachsenenbildung) (2004). Nationale Bildungsstandards für den Fachunterricht in der Politischen Bildung an Schulen. Ein Entwurf. Schwalbach/Ts.: Wochenschau.

Grammes, Tilmann (2014). Kontroversität. In Wolfgang Sander (Hrsg.), Handbuch Politische Bildung. Schwalbach/Ts.: Wochenschau, S. 266-274.

Hafeneger, Benno (2017). Aufklärung, Wissen und Reflexion vs. Fake News, Mythen und Legenden. Herausforderungen für die politische Bildung. Journal für politische Bildung, (4), S. 48-53.

Henkenborg, Peter (2013). Überlegungen zu einer kritischen Theorie politischer Bildung. In Benedikt Widmaier \& Bernd Overwien (Hrsg.), Was heißt heute kritische politische Bildung? Schwalbach/Ts.: Wochenschau, S. 109-118.

Hippe, Thorsten (2016). Herausforderung Klimaschutzpolitik. Probleme, Lösungsstrategien und Kontroversen. Opladen: Barbara Budrich.

Hippe, Thorsten (2018). Kritik der gesellschaftlichen Lebenslüge. Zeitschrift für Didaktik der Gesellschaftswissenschaften (1), S. 21-42.

Hufer, Klaus-Peter (2013). Politische Bildung aus dem Geist der Aufklärung: Ein Grundverständnis und seine Herleitung. In ders., Theo W. Länge, Barbara Menke, Bernd Overwien, \& Laura Schudoma (Hrsg.), Wissen und Können: Wege zum professionellen Handeln in der politischen Bildung. Schwalbach/Ts.: Wochenschau, S. 23-34.

Kenner, Steve (2021). Politische Bildung in Aktion. Von der Antirassismus-Initiative bis „Fridays for Future“. Eine qualitative Studie zu Bildungserfahrungen von Schüler*innen in selbstbestimmter politischer Partizipation. Hannover: Leibnitz-Universität (im Erscheinen).

Klafki, Wolfgang (1996). Neue Studien zur Bildungstheorie und Didaktik. Zeitgemäße Allgemeinbildung und kritisch-konstruktive Didaktik (4. Aufl.). Weinheim: Beltz.

Klafki, Wolfgang (2005). Allgemeinbildung in der Grundschule und der Bildungsauftrag des Sachunterrichts. Widerstreit Sachunterricht (4).

http://www.widerstreit-sachunterricht.de/ebeneII/arch/klafki/klafki.htm

KMK (Kultusministerkonferenz)/BMZ (Bundesministeriums für wirtschaftliche Zusammenarbeit und Entwicklung) (2016). Orientierungsrahmen für den Lernbereich Globale Entwicklung im Rahmen einer Bildung für nachhaltige Entwicklung (2. Auf.). Berlin. http://www.orientierungsrahmen.de 


\section{Bernd Overwien}

Mittnik, Philipp; Lauss, Georg; Schmid-Heher, Stefan (2018). Was darf politische Bildung? Eine Handreichung für LehrerInnen für den Unterricht in Politischer Bildung. Wien: Pädagogische Hochschule. https://zpb.phwien.ac.at/wp-content/uploads/Was_darf_politische_Bildung_A4.pdf

Nonnenmacher, Frank (2011). Handlungsorientierung und politische Aktion in der schulischen politischen Bildung. Ursprünge, Grenzen und Herausforderungen. In Benedikt Widmaier \& Frank Nonnenmacher (Hrsg.), Partizipation als Bildungsziel. Politische Aktion in der politischen Bildung. Schwalbach/Ts.: Wochenschau, 83-110.

Oberle, Monika (2017). Wie politisch dürfen, wie politisch sollen Politiklehrer/-innen sein? Politische Orientierung von Lehrkräften als Element ihrer Professionellen Kompetenz. In Siegfried Frech \& Dagmar Richter (Hrsg.), Der Beutelsbacher Konsens. Bedeutung, Wirkung, Kontroversen. Schwalbach/ Ts.: Wochenschau, S. 114-127.

Overwien, Bernd (2005). Stichwort: Informelles Lernen. ZfE 8(3), S. 339-355.

Overwien, Bernd (2016). Der Beutelsbacher Konsens im Kontext Globalen Lernens und der Bildung für nachhaltige Entwicklung. In Benedikt Widmaier \& Peter Zorn (Hrsg.), Brauchen wir den Beutelsbacher Konsens? Eine Debatte der politischen Bildung. Bonn: BpB, S. 260-268.

Overwien, Bernd (2017). Politische Bildung und Globales Lernen: Distanz zwischen Wissenschaft und Praxis? In Oliver Emde, Uwe Jakubczyk, Bernd Kappes \& Bernd Overwien (Hrsg.), Mit Bildung die Welt verändern? Globales Lernen für eine nachhaltige Entwicklung. Opladen: Barbara Budrich, S. $121-132$.

Overwien, Bernd (2019). Umwelt, Klimawandel, Globalisierung - Angst in der politischen Bildung? In Anja Besand, Bernd Overwien \& Peter Zorn (2019), Politische Bildung mit Gefühl. Bonn: BpB, S. 304-318.

Reinhardt, Sibylle (2017). Wie politisch darf eine Politiklehrkraft sein? In Sabine Achour \& Thomas Gill (Hrsg.), Was politische Bildung alles kann. Einführung in die politische Bildung. Schwalbach/Ts.: Wochenschau, S. 105-114.

Sander, Wolfgang (2013). Politik in der Schule. Kleine Geschichte der politischen Bildung in Deutschland (3. akt. Aufl.). Marburg: Schüren.

Sander, Wolfgang (2014). Kompetenzorientierung als Forschungs- und Konfliktfeld der Didaktik der politischen Bildung. In Wolfgang Sander (Hrsg.), Handbuch politische Bildung. SchwalbachTs.: Wochenschau, S. 113-126.

Schmiederer, Rolf (1971). Zur Kritik der politischen Bildung. Ein Beitrag zur Soziologie und Didaktik des Politischen Unterrichts. Frankfurt a. M.: Europäische Verlagsanstalt.

Schneekloth, Ulrich \& Albert, Mathias (2010). Entwicklungen bei den „großen Themen“: Generationengerechtigkeit, Globalisierung, Klimawandel. In Mathias Albert, Klaus Hurrelmann \& Gudrun Quenzel (Hrsg.), Jugend 2010. Shell-Jugendstudie. Frankfurt a. M.: Fischer, S. 165-184.

Singer-Brodowski, Mandy (2016). Transformative Bildung durch transformatives Lernen. Zur Notwendigkeit der erziehungswissenschaftlichen Fundierung einer neuen Idee. Zeitschrift für internationale Bildungsforschung und Entwicklungspädagogik 39(1), S. 13-17.

Sliwka, Anne (2004). Durch Verantwortung lernen. Service-Learning: etwas für andere tun. Weinheim/ Basel: Beltz.

WBGU (Wissenschaftlicher Beirat der Bundesregierung Globale Umweltveränderungen) (2011). Welt im Wandel - Gesellschaftsvertrag für eine Große Transformation. Hauptgutachten. Berlin.

Wohnig, Alexander (2017). Zum Verhältnis von sozialem und politischem Lernen. Eine Analyse von Praxisbeispielen politischer Bildung. Wiesbaden: Springer.

Wohnig, Alexander (2018). Demokratiebildung durch politische Aktion. In Steve Kenner \& Dirk Lange (Hrsg.), Citizenship Education. Konzepte, Anregungen und Ideen zur Demokratiebildung. Frankfurt a. M.: Wochenschau. S. 269-281. 